\title{
Functional innervation imaging in the evaluation of cardiotoxicity: Just the beginning of the journey
}

\author{
Matteo Bauckneht, MD, PhD (1), and Gianmario Sambuceti, $M D^{\mathrm{a}, \mathrm{b}}$ \\ a Nuclear Medicine, IRCCS Ospedale Policlinico San Martino, Genoa, Italy \\ b Department of Health Sciences, University of Genoa, Genoa, Italy
}

Received May 4, 2021; accepted May 4, 2021

doi: 10.1007/s12350-021-02668-w

\section{See related article, pp. 2274-2291}

Treatment effectiveness for solid and hematological tumors significantly improved over the last decades. However, among the growing number of cancer survivors, it has been documented a notable increase in the prevalence of chemotherapy-related side effects, whose incidence rises with elapsed time for months and years after treatment completion. This phenomenon is particularly evident in patients treated with chemotherapy schemes containing anthracyclines, in whom left ventricular (LV) dysfunction may occur in up to $48 \%$ of cases in a dose-dependent manner. ${ }^{1}$

In this scenario, one of the emerging clinical issues involves the silent occurrence of asymptomatic LV dysfunction. In these cases, a good functional recovery can be achieved only if the cardiac damage is early detected and prompt treated with medications. ${ }^{2}$ Conversely, if patients are identified late after the onset of cardiac dysfunction, heart failure is typically difficult to treat. $^{2}$ On these bases, several research efforts have been dedicated to identifying early non-invasive detection strategies.

Current expert consensus recommends using cardiac imaging to identify subtle changes of the LV ejection fraction (LVEF) during and after chemotherapy. $^{2,3}$ Serial natriuretic peptides and troponins measurements may also be considered, depending on

Reprint requests: Matteo Bauckneht, MD, PhD, Nuclear Medicine, IRCCS Ospedale Policlinico San Martino, Largo Rosanna Benzi 10, 16132 Genoa, Italy; matteo.bauckneht@gmail.com

J Nucl Cardiol 2022;29:2292-4.

1071-3581/ $\$ 34.00$

Copyright (c) 2021 American Society of Nuclear Cardiology. local expertise and availability. ${ }^{2,3}$ However, in the initial phases of cardiac injury, this gold-standard approach lacks in sensitivity, ${ }^{4}$ as it interrogates the late consequences of the cardiotoxic damage rather than its (intrinsically earlier) pathophysiological determinants.

Nuclear medicine represents a natural candidate to interrogate cardiotoxicity at a molecular level, potentially identifying cardiac diseases not detected by clinical criteria or conventional methods. In the last years, a few studies suggested that the generation of reactive oxygen species induced by anthracyclines can be tracked in vivo by ${ }^{18}$ F-Fluorodeoxyglucose uptake ${ }^{5}$ as an early, subclinical sign of the subsequent contractile dysfunction. ${ }^{6}$ Similarly, given the adrenergic damage induced by anthracyclines, ${ }^{7,8}$ the non-invasive detection of adrenergic dysfunction using Iodine-123 metaiodobenzylguanidine $\left({ }^{123} \mathrm{I}-\mathrm{mIBG}\right)$ scintigraphy ${ }^{9}$ may represent a useful tool for the early detection of cardiotoxicity.

In the current issue of the Journal of Nuclear Cardiology, Arrais et al. propose a systematic review to evaluate the performance of cardiac innervation imaging using ${ }^{123} \mathrm{I}$-mIBG in this clinical context. ${ }^{10}$ As underlined by the authors, available studies can be divided in three main categories including (1) studies comparing patients treated with anthracyclines compared to controls; (2) studies enrolling patients with established cardiotoxicity, and (3) studies focused on the anthracycline's doserelated changes of ${ }^{123} \mathrm{I}-\mathrm{mIBG}$ parameters. The lowest common denominator of these clinical scenarios seems to be the usefulness of the delayed heart-to-mediastinum ratio (dHMR). Indeed, dHMR is significantly reduced in chemotherapy-treated patients vs controls (even in the presence of normal LVEF), mean dMHR is below 1.7 in all studies enrolling patients with established cardiotoxicity, and the degree of its reduction is inversely related to the cumulative dose of administered. The adrenergic damage is additionally expressed in patients 
receiving trastuzumab in addition to anthracyclines. Though not explicitly addressed by Arrais et al., ${ }^{10}$ taking into account the burgeoning literature on the prognostic value of ${ }^{123} \mathrm{I}-\mathrm{mIBG}$ in heart failure, we can also assume that the anthracycline-related myocardial adrenergic impairment might also stratify the long-term prognosis of cancer survivors.

Reported findings unquestionably suggest a potential role for ${ }^{123}$ I-mIBG scintigraphy in the field of cardio-oncology. However, as underlined by the authors, the few and heterogeneous studies make it difficult to extrapolate robust conclusions, and further larger prospective studies are needed in the next future to corroborate obtained results. Nevertheless, (even) before planning validating large-scale studies, several issues (mainly methodological) still remain to be addressed. In particular, the standardization of image acquisition and analyses are mandatory. In the included studies, ${ }^{123} \mathrm{I}-$ mIBG derived parameters were mainly assessed manually (and thus operator-dependently), drawing regions of interest over the heart and the upper mediastinum in the planar anterior view. Furthermore, low energy and medium energy collimators equipping Anger cameras were indiscriminately used, thus potentially resulting in different normal and pathologic HMR values. ${ }^{11,12}$ Therefore, the major challenge before planning to validate the use of ${ }^{123} \mathrm{I}$-mIBG imaging in cardio-oncology is harmonizing the imaging technology. The validation of advanced analytic tools and the shift from planar to tomographic imaging will be part of this process, which will be even more complicated in the coming years, given the growing spreading of dedicated cardiac gamma-cameras with multi-focus collimator and cadmium-zinc-telluride, able to generate tomographic images only. ${ }^{13}$

Not less important are the fields of radiation exposure and costs. Given the radiation-free nature of the current imaging competitors in cardio-oncology (namely echocardiography and cardiac magnetic resonance imaging), ${ }^{123}$ I-mIBG imaging needs to be proved to provide superior diagnostic or prognostic data to avoid unnecessary radiation exposure. Similarly, the cost-effectiveness of this procedure in cardio-oncology needs to be investigated, as already performed with contradicting results in neurology and cardiology. ${ }^{14,15}$ In order to achieve these aims, ${ }^{123}$ I-mIBG needs to be compared to competitors in individuals belonging to different risk classes for the later development of cardiotoxicity (e.g., based on the cumulative received anthracycline dose). This may clarify whether individuals at greater cardiotoxic risk are more likely to benefit from the supplementary imaging modality.

As a final consideration, although the current evidence is promising, the future use of ${ }^{123} \mathrm{I}-\mathrm{mIBG}$ scintigraphy in cardio-oncology will most likely depend on its translation to daily clinical practice. In the current era, to drive the implementation of a diagnostic tool into daily practice, it is mandatory to show its capability to improve the clinical outcome. Therefore, once explored more sophisticated semi-quantification tools, harmonized acquisition processes, incorporated advanced technologies, and selected patients most likely to benefit from ${ }^{123} \mathrm{I}-\mathrm{mIBG}$ imaging, this implementation roadmap will need to assess its capability to change the patient outcome (e.g., by personalizing risk-stratification and allowing prompt intervention to minimize or prevent the irreversible cardiac damage). In other words, we are just at the beginning of the journey. However, to say it with Plato "the beginning is the most important part of the work".

\section{Disclosures}

None.

\section{References}

1. Ameri P, Canepa M, Anker MS, Belenkov Y, Bergler-Klein J, Cohen-Solal A, et al. Cancer diagnosis in patients with heart failure: Epidemiology, clinical implications and gaps in knowledge. Eur J Heart Fail. 2018;20:879-87.

2. Zamorano JL, Lancellotti P, Rodriguez Muñoz D, Aboyans V, Asteggiano R, et al. 2016 ESC Position Paper on cancer treatments and cardiovascular toxicity developed under the auspices of the ESC Committee for Practice Guidelines: The Task Force for cancer treatments and cardiovascular toxicity of the European Society of Cardiology (ESC). Eur J Heart Fail. 2017;19:9-42.

3. Plana JC, Galderisi M, Barac A, Ewer MS, Ky B, Scherrer-Crosbie $\mathrm{M}$, et al. Expert consensus for multimodality imaging evaluation of adult patients during and after cancer therapy: A report from the American Society of Echocardiography and the European Association of Cardiovascular Imaging. J Am Soc Echocardiogr. 2014;27:911-39.

4. Sawaya H, Sebag IA, Plana JC, Januzzi JL, Ky B, Tan TC, et al. Assessment of echocardiography and biomarkers for the extended prediction of cardiotoxicity in patients treated with anthracyclines, taxanes, and trastuzumab. Circ Cardiovasc Imaging. 2012;5:596603.

5. Bauckneht M, Pastorino F, Castellani P, Cossu V, Orengo AM, Piccioli P, et al. Increased myocardial 18F-FDG uptake as a marker of Doxorubicin-induced oxidative stress. J Nucl Cardiol. 2020;27:2183-94.

6. Sarocchi M, Bauckneht M, Arboscello E, Capitanio S, Marini C, Morbelli S, et al. An increase in myocardial 18-fluorodeoxyglucose uptake is associated with left ventricular ejection fraction decline in Hodgkin lymphoma patients treated with anthracycline. J Transl Med. 2018;16:295.

7. Valdés Olmos RA, ten Bokkel Huinink WW, ten Hoeve RF, van Tinteren H, Bruning PF, van Vlies B, et al. Assessment of anthracycline-related myocardial adrenergic derangement by [123I]metaiodobenzylguanidine scintigraphy. Eur J Cancer. 1995;31A:26-31. 
8. Ewer MS, Lenihan DJ. Left ventricular ejection fraction and cardiotoxicity: Is our ear really to the ground? J Clin Oncol. 2008;26:1201-3.

9. Bauckneht M, Sambuceti G, Pomposelli E, Fiz F, Marini C. Pathophysiological basis of myocardial innervation imaging in heart failure. Clin Transl Imaging. 2015;3:347-55.

10. Arrais et al. J Nucl Cardiol xxxx, yy, zz-zz in press

11. Veltman CE, Boogers MJ, Meinardi JE, Al Younis I, DibbetsSchneider P, Van der Wall EE, et al. Reproducibility of planar (123)I-meta-iodobenzylguanidine (mIBG) myocardial scintigraphy in patients with heart failure. Eur J Nucl Med Mol Imaging. 2012;39:1599-608.

12. Verberne HJ, Feenstra C, de Jong WM, Somsen GA, van Eck-Smit BLF, Busemann SE. Influence of collimator choice and simulated clinical conditions on 123I-mIBG heart/mediastinum ratios: A phantom study. Eur J Nucl Med Mol Imaging. 2005;32:1100-7.

13. Shibutani T, Nakajima K, Yoneyama H, Konishi T, Okuda K, Onoguchi $\mathrm{M}$, et al. The utility of heart-to-mediastinum ratio using a planar image created from IQ-SPECT with Iodine-123 metaiodobenzylguanidine. J Nucl Cardiol. 2020. https://doi.org/10.10 07/s12350-020-02081-9.

14. Ikeda T, Ikenoshita S, Sakamoto F, Shiraishi S, Nakahara K, Masuda T, et al. Is 123I-MIBG scintigraphy beneficial or excessive for the diagnosis of Parkinson's disease in the early phase? Neurodegener Dis. 2019;19:88-95.

15. O’Day K, Levy WC, Johnson M, Jacobson AF. Cost-effectiveness analysis of iodine-123 meta-iodobenzylguanidine imaging for screening heart failure patients eligible for an implantable cardioverter defibrillator in the USA. Appl Health Econ Health Policy. 2016;14:361-73.

Publisher's Note Springer Nature remains neutral with regard to jurisdictional claims in published maps and institutional affiliations. 